\title{
COMET: visualisation of regional epigenome-wide association scan results and DNA co-methylation patterns
}

\author{
Tiphaine C Martin ${ }^{*}$, Idil Yet, Pei-Chien Tsai and Jordana T Bell ${ }^{*}$
}

\begin{abstract}
Background: Epigenome-wide association scans (EWAS) are an increasingly powerful and widely-used approach to assess the role of epigenetic variation in human complex traits. However, this rapidly emerging field lacks dedicated visualisation tools that can display features specific to epigenetic datasets.

Result: We developed coMET, an R package and online tool for visualisation of EWAS results in a genomic region of interest. CoMET generates a regional plot of epigenetic-phenotype association results and the estimated DNA methylation correlation between $\mathrm{CpG}$ sites (co-methylation), with further options to visualise genomic annotations based on ENCODE data, gene tracks, reference CpG-sites, and user-defined features. The tool can be used to display phenotype association signals and correlation patterns of microarray or sequencing-based DNA methylation data, such as Illumina Infinium 450k, WGBS, or MeDIP-seq, as well as other types of genomic data, such as gene expression profiles. The software is available as a user-friendly online tool from http://epigen.kcl.ac.uk/comet and as an $\mathrm{R}$ Bioconductor package. Source code, examples, and full documentation are also available from GitHub.

Conclusion: Our new software allows visualisation of EWAS results with functional genomic annotations and with estimation of co-methylation patterns. cOMET is available to a wide audience as an online tool and R package, and can be a valuable resource to interpret results in the fast growing field of epigenetics. The software is designed for epigenetic data, but can also be applied to genomic and functional genomic datasets in any species.
\end{abstract}

Keywords: Epigenome-wide association scan, EWAS, DNA methylation, Co-methylation, Visualisation, Gene expression, Functional annotation, Bioconductor

\section{Background}

Epigenome-wide association studies (EWAS) systematically test for association between DNA methylation variation and human complex traits [1,2]. Recent EWAS have identified differentially methylated regions (DMRs) and variably methylated regions (VMRs) for multiple phenotypes, diseases, and environmental exposures (for example, [3-7]) and many on-going efforts are currently underway. A major challenge lies in interpreting EWAS signals. One problem is defining the exact region of association, for example, distinguishing between a single differentially methylation position (DMP) and a differentially methylated region (DMR) containing multiple DMPs.

*Correspondence: tiphaine.martin@kcl.ac.uk; jordana.bell@kcl.ac.uk Department of Twin Research and Genetic Epidemiology, St Thomas' Hospital Campus, King's College London, Westminster Bridge Road, London, UK
DNA methylation levels at nearby CpG-sites (within 2kb apart) can be highly correlated [8,9]. Analyzing clusters of co-methylated CpG sites may be more informative than single-CpG analysis [9], yet from a functional perspective identifying specific $\mathrm{DMP}(\mathrm{s})$ with potential molecular consequences is critical. A number of recent packages including Bumphunter [10], Minfi [11], ChAMP [12], Aclustering [13], and RnBeads [14] can identify DMRs, but none of these methods allow the visualization of the correlation between DMPs within a DMR. On the other hand, several R packages, including snp.plotter [15] and LocusZoom [16] have been developed to visualise GWAS association results and linkage disequilibrium (LD) patterns, but none exists for EWAS. At present, EWAS datasets typically consist of quantitative levels of DNA methylation 
at different sites or regions, where each sample represents a population of cells from an individual. These data are therefore unsuitable for standard LD plots and would benefit from dedicated user-friendly methylation plotting tools.

EWAS findings can provide mechanistic insights into disease susceptibility or progression, and should be explored in functional genomic context. A comparison across multiple layers of epigenetic marks and chromatin domains can help define the functional context of a genomic region. Several R packages, such as Gviz [17], trackViewer [18], ggbio [19], GenomeGraphs [20], or methyAnalysis [21], can help us to visually explore different annotation tracks in genomic regions. However, most of these packages do not have options to concurrently visualise phenotype-association results or co-methylation patterns at $\mathrm{CpG}$-sites or regions.

We have developed coMET, an $\mathrm{R}$ package and webbased tool to generate regional plots of EWAS data and results. coMET can be applied to visualise regional EWAS results from analyses of both single-CpG and regionbased datapoints, for example using microarray-based technologies (such as Illumina 450k) or sequencing-based methods (such as whole genome bisulfite sequencing (WGBS) or DNA methylation capture by immunoprecipitation followed by sequencing (MeDIP-seq)), and to compute and visualise also the correlations between the CpG-sites or regions. The web-service [22] allows users to run a pre-formatted version of coMET.

\section{Implementation}

coMET is implemented in $\mathrm{R}$ to produce a multi-panel plot to visualise EWAS results, genomic annotations, and to estimate and plot DNA co-methylation patterns. The structure of the plots builds on snp.plotter [15], with extensions to incorporate genomic annotation tracks and customized functions. Plots can be generated in PDF or EPS format. coMET is available as an $R$ package or as a web-service.

\section{coMET R package}

The coMET $\mathrm{R}$ package is available for download from Bioconductor [23] or online from GitHub [24]. The package includes source code, two sample datasets, documentation and vignette. Analysis requires $\mathrm{R}$ version 3.1.1 or higher and an active internet connection to enable direct download of up to date annotation tracks from Ensembl mart databases [25] and UCSC [26]. The coMET R Bioconductor package has currently two main functions: 'comet.web' and 'comet'. The function 'comet.web' generates output plots with the predefined genomic annotation track settings used for the web-service and the function 'comet' generates output plots with customised annotation tracks defined by the user.

\section{cOMET web-service}

The coMET website [22] allows users to run a preformatted version of coMET. The web-service is developed in Shiny [27] and can be installed locally on a machine running $\mathrm{R}$ version 3.1.1 or higher, Bioconductor version 3.1 or higher and Shiny. It requires at least 4GB of memory and at least 10GB of available disk space. The web-service also requires an active internet connection to download up to date annotation tracks.

\section{Data input}

Data input for coMET includes a data file or matrix describing EWAS results at the DNA methylation CpGsites or genomic regions to visualise ("mydata.file" and "mydata.type"), a data file or matrix describing the co-methylation dataset ("cormatrix.file" and "cormatrix.type"), and a configuration file containing the plot parameters ("config.file"). Currently, coMET can visualise the correlation of a maximum of 120 pre-defined features on the plot, due to limitations on the size of the plot. Optional input files can also be uploaded to include association P-values from a larger genomic region of interest in the upper plot or to include them as user-defined annotation tracks in the middle panel, but the lower panel is limited to visualizing only up to $120 \mathrm{CpG}$-sites or regions which can represent a subset of the larger genomic region in the top panel if required (see example in Figure 1).

\section{Results and discussion}

coMET generates a multi-panel plot to visualise EWAS results, co-methylation patterns, and annotation tracks in a genomic region of interest. A coMET figure (Figure 1) includes three components: (1) the upper plot shows the strength and extent of EWAS association signal; (2) the middle panel provides customized annotation tracks; and (3) the lower panel shows the co-methylation between selected $\mathrm{CpG}$ sites in the genomic region.

\section{EWAS signal}

The top panel of the coMET plot shows EWAS phenotype association P-values on a $-\log 10$ scale according to chromosomal position. The user can specify the region of interest in the input dataset and zoom in/out to view a subset of the region. A reference CpG-site can be highlighted in the association plot, using userspecified colours. The association plot can also optionally denote the direction of phenotype-association using colour-coded symbols. If region-based rather than singleCpG datapoints are visualised the plotting symbols can also denote the size of the regional unit of analysis.

\section{Annotation tracks}

The middle panel includes optional genomic annotation tracks, for example, functional annotations from Ensembl, 


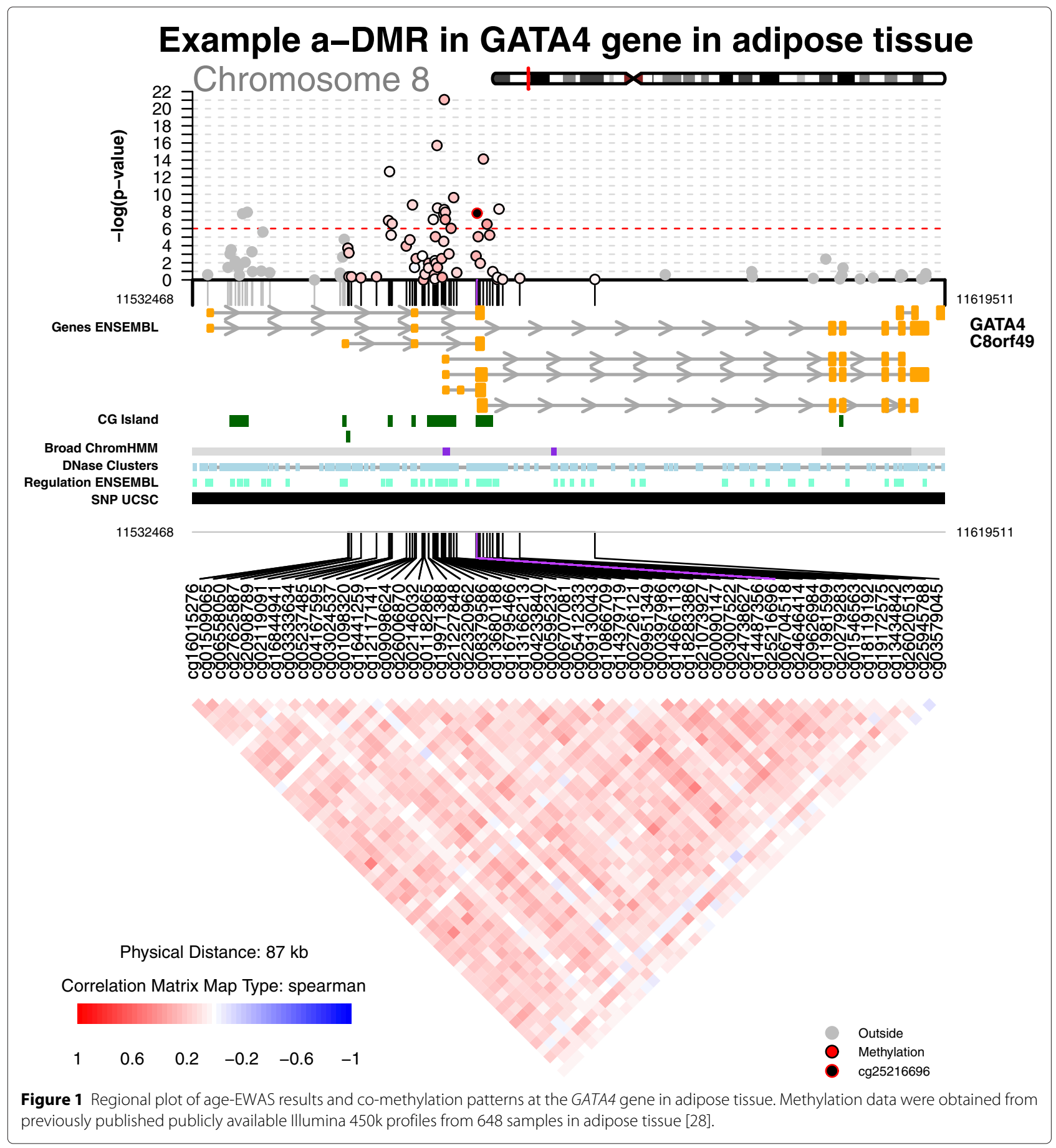

ENCODE and UCSC for any species and versions available such as hg38, hg19 or GRCm38. By default, the pre-formatted version of coMET ('comet.web') includes six optional annotation tracks: genes or transcripts (Ensembl), CpG islands (UCSC), Broad ChromHMM domains (UCSC), DNaseI clusters (UCSC), Ensembl regulation tracks, and SNPs (UCSC). These tracks are obtained directly from the revelant online server or data repository
(Ensembl BioMart or UCSC tracks) at the time of analysis. This allows for visualisation of up to date functional annotation data, but the analysis requires an active internet connection. In addition to the optional pre-defined tracks, user-defined annotation and data tracks can also be included in a format accepted by Gviz. Altogether, up to 6 annotation tracks can be viewed in the basic version of the package. The generic version of coMET ('comet') can 
visualise lists of customized annotation and data tracks using Gviz [17], ggbio [19], and trackViewer [18].

\section{Co-methylation}

The lower panel represents the correlation in DNA methylation levels between selected CpG-sites in the genomic region, or co-methylation. The correlation matrix and the significant of correlations are calculated based on user-provided DNA methylation values (e.g. beta values for the Illumina 450k array) for selected CpG-sites or regions, the selected correlation method (Spearman, Pearson, Kendall), and the selected alpha level for the confidence interval (e.g. alpha $=0.05$ for $95 \% \mathrm{CI}$ ). A userprovided correlation matrix can also be used. The colour scheme of the heatmap represents the correlation scale (for example, from -1 (blue) to 1 (red) in Figure 1) and can be reflected in the top association panel of the coMET plot with respect to correlation to the user-defined reference CpG site.

\section{Examples}

To show the functionality of coMET, we explored previously published Illumina 450k DNA methylation profiles from adipose tissue in 648 individuals with available age at biopsy [28]. We first performed an EWAS of chronological age and found strong age association with the previously identified age differentially methylation region (a-DMR) in the GATA4 gene, which is an a-DMR in whole blood, muscle, kidney, and brain samples [29,30]. Figure 1 shows age association results at $95 \mathrm{CpG}$ sites (upper plot), with 6 default annotation tracks, and estimated co-methylation patterns at 55 selected $\mathrm{CpG}$ sites (lower panel) in the GATA4 gene on chromosome 8 . The results are shown with respect to reference CpG site cg25216696, which is the most associated a-DMP in our data and in previously published whole blood datasets. These findings show for the first time that the GATA4 a-DMR is present in adipose tissue.

Recently, Richmond et al. [31] also used the online version of coMET to visualise differential methylation and co-methylation patterns of 7 genomic regions using Illumina $450 \mathrm{k}$ data.

\section{Conclusion}

coMET is a user-friendly R-package and online tool that allows for quick and flexible visualisation of EWAS results, co-methylation patterns, and functional annotation. The software is designed for epigenetic data, but can also be applied to genomic and functional genomic datasets in any species.

\author{
Availability and requirements \\ Project name: coMET \\ Project home page: http://epigen.kcl.ac.uk/comet
}

Operating system(s): Platform independent Programming language: $\mathrm{R} 3.1 .1$ or higher Other requirements: Bioconductor 3.1 or higher, Shiny License: GNU GPL 2 or higher

Any restrictions to use by non-academics: none

\section{Competing interests}

The authors declare that they have no competing interests.

\section{Authors' contributions}

JTB conceived the project. TCM designed and implemented the project with help from IY and PCT. TCM and JTB wrote the manuscript. All authors read and approved the final manuscript.

\section{Acknowledgements}

The authors thank Craig Glastonbury for testing coMET, and Kai Fong Lu, Joan Bryan and Greg McGarrick from the KCL IT team for creating the virtual machine to host the R shiny web-service.

\section{Funding}

The study received support from the Wellcome Trust (082713/Z/07/Z), the European Research Council (ERC 250157), and the TwinsUK resource, which is funded by the Wellcome Trust and the European Community's Seventh Framework Programme (FP7/2007-2013), with support from the National Institute for Health Research (NIHR) - funded BioResource, Clinical Research Facility and Biomedical Research Centre based at Guy's and St Thomas' NHS Foundation Trust in partnership with King's College London.

Received: 28 November 2014 Accepted: 10 April 2015

Published online: 28 April 2015

\section{References}

1. Petronis A. Epigenetics as a unifying principle in the aetiology of complex traits and diseases. Nature. 2014:465(7299):721-7.

2. Rakyan VK, Down TA, Balding DJ, Beck S. Epigenome-wide association studies for common human diseases. Nat Rev Genet. 2011;12(8):529-41.

3. Jaffe AE, Feinberg AP, Irizarry RA, Leek JT. Significance analysis and statistical dissection of variably methylated regions. Biostatistics. 2012;13(1):166-78.

4. Liu Y, Aryee MJ, Padyukov L, Fallin MD, Hesselberg E, Runarsson A, et al. Epigenome-wide association data implicate DNA methylation as an intermediary of genetic risk in rheumatoid arthritis. Nat Biotechnol. 2013;31(2):142-7.

5. Bell JT, Loomis A, Butcher L, Gao ZBF, Hyde CL, Sun J, et al. Differential methylation of the TRPA1 promoter in pain sensitivity. Nat Commun. 2014;5:2978.

6. Dominguez-Salas P, Moore SE, Baker MS, Bergen AW, Cox SE, Dyer RA et al. Maternal nutrition at conception modulates DNA methylation of human metastable epialleles. Nature Commun. 2014;5:3746.

7. Ong ML, Holbrook JD. Novel region discovery method for infinium 450k DNA methylation data reveals changes associated with aging in muscle and neuronal pathways. Aging Cell. 2014;13(1):142-55.

8. Bell JT, Pai AA, Pickrell JK, Gaffney DJ, Pique-Regi R, Degner JF, et al. DNA methylation patterns associate with genetic and gene expression variation in hapmap cell lines. Genome Biol. 2011;12(1):10.

9. Liu Y, Li X, Aryee MJ, Ekström TJ, Padyukov L, Klareskog L, et al. Gemes, clusters of DNA methylation under genetic control, can inform genetic and epigenetic analysis of disease. Am J Hum Genet. 2014;94(6):485-95.

10. Jaffe AE, Murakami $P$, Lee $H$, Leek JT, Fallin MD, Feinberg AP, et al. Bump hunting to identify differentially methylated regions in epigenetic epidemiology studies. Int J Epidemiol. 2012;41(1):200-9.

11. Aryee MJ, Jaffe AE, Corrada-Bravo H, Ladd-Acosta C, Feinberg AP, Hansen KD, et al. Minfi: a flexible and comprehensive Bioconductor package for the analysis of infinium DNA methylation microarrays. Bioinformatics. 2014;15(30):10.

12. Morris TJ, Butcher LM, Feber A, Teschendorff AE, Chakravarthy AR, Wojdacz TK, et al. ChAMP: 450k chip analysis methylation pipeline. Bioinformatics. 2014:30(1):428-30. 
13. Sofer T, Schifano ED, Hoppin JA, Hou L, Baccarelli AA. A-clustering: a novel method for the detection of co-regulated methylation regions, and regions associated with exposure. Bioinformatics. 2013;29(22):2884-91.

14. Assenov $Y$, Müller F, Lutsik P, Walter J, Lengauer T, Bock C Comprehensive analysis of DNA methylation data with RnBeads. Nat Methods. 2014;11(11):1138-40.

15. Luna A, Nicodemus KK. snp.plotter: an R-based snp/haplotype association and linkage disequilibrium plotting package. Bioinformatics. 2014;23(6):774-6.

16. Pruim RJ, Welch RP, Sanna S, Teslovich TM, Chines PS, Gliedt TP, et al. LocusZoom: Regional visualization of genome-wide association scan results. Bioinformatics. 2010;26(18):2336-7.

17. Hahne F, Durinck S, Ivanek R, Mueller A, Lianoglou S, Tan G. Gviz: Plotting data and annotation information along genomic coordinates. Bioconductor. 2015. http://bioconductor.org/packages/release/bioc/ html/Gviz.html. R package version 1.12.0. Accessed 23 April 2015.

18. Wang Y, Zhu LJ. trackViewer: A Bioconductor package with minimalist design for plotting elegant track layers. Bioconductor. 2015. http:// bioconductor.org/packages/release/bioc/html/trackViewer.html. R package version 1.4. Accessed 23 April 2015.

19. Yin T, Cook D, Lawrence M. ggbio: an R package for extending the grammar of graphics for genomic data. Genome Biol. 2012;13(8):R77.

20. Durinck S, Bullard J, Spellman PT, Dudoit S. GenomeGraphs: integrated genomic data visualisation with R. BMC Bioinformatics. 2009;10:2.

21. Du P, Bourgon R. MethyAnalysis: DNA methylation data analysis and visualization. Bioconductor. 2015. http://bioconductor.org/packages/ release/bioc/html/methyAnalysis.html. R package version 1.10.0. Accessed 23 April 2015

22. Martin TC, Yet I, Tsai PC, Bell JT. coMET Website. 2015. http://epigen.kcl. ac.uk/comet. Accessed 23 April 2015

23. Huber W, Carey VJ, Gentleman R, Anders S, Carlson M, Carvalho BS, et al. Orchestrating high-throughput genomic analysis with Bioconductor. Nat Methods. 2015;12(2):115-21. http://bioconductor.org/packages/ release/bioc/html/coMET.html.

24. Martin TC, Yet I, Tsai PC, Bell JT. coMET GitHub. 2015. https://github. com/TiphaineCMartin/coMET. Accessed 23 April 2015.

25. Ensembl: BioMart - Ensembl. 2015. http://www.ensembl.org/biomart/ martview/. Accessed 23 April 2015.

26. UCSC Genome Bioinformatics: UCSC Genome Browser Home. 2015. https://genome.ucsc.edu. Accessed 23 April 2015.

27. RStudio: Shiny. 2015. http://shiny.rstudio.com/. Accessed 23 April 2015.

28. Grundberg E, Meduri E, Sandling JK, Hedman AK, Keildson S, Buil A, et al. Global analysis of DNA methylation variation in adipose tissue from twins reveals links to disease-associated variants in distal regulatory elements. Am J Hum Genet. 2013;93(5):876-90.

29. Day K, Waite LL, Thalacker-Mercer A, West A, Bamman MM, Brooks JD, et al. Differential DNA methylation with age displays both common and dynamic features across human tissues that are influenced by $\mathrm{CpG}$ landscape. Genome Biol. 2013;14(9):102.

30. Johansson A, Enroth S, Gyllensten U. Continuous aging of the human DNA methylome throughout the human lifespan. PLOS ONE. 2013;8(6): 67378.

31. Richmond RC, Simpkin AJ, Woodward G, Gaunt TR, Lyttleton O, McArdle WL, et al. Prenatal exposure to maternal smoking and offspring DNA methylation across the lifecourse: findings from the Avon Longitudinal Study of Parents And Children (ALSPAC). Hum Mol Genet. 2015;24:2201-17.

\section{Submit your next manuscript to BioMed Central and take full advantage of:}

- Convenient online submission

- Thorough peer review

- No space constraints or color figure charges

- Immediate publication on acceptance

- Inclusion in PubMed, CAS, Scopus and Google Scholar

- Research which is freely available for redistribution 